\title{
Dynamic transitions in a model of the hypothalamic-pituitary-adrenal axis
}

\author{
Željko Čupić, ${ }^{1, a)}$ Vladimir M. Marković, ${ }^{2}$ Stevan Maćešić, ${ }^{2}$ Ana Stanojević, ${ }^{2}$ \\ Svetozar Damjanović, ${ }^{3}$ Vladana Vukojević, ${ }^{4}$ and Ljiljana Kolar-Anić ${ }^{1,2}$ \\ ${ }^{1}$ Department of Catalysis and Chemical Engineering, Institute of Chemistry, Technology and Metallurgy, \\ University of Belgrade, Njegoševa 12, 11000 Belgrade, Serbia \\ ${ }^{2}$ Faculty of Physical Chemistry, University of Belgrade, Studentski trg 12-16, 11158 Belgrade, Serbia \\ ${ }^{3}$ Institute of Endocrinology, Diabetes and Metabolic Diseases, School of Medicine, University of Belgrade, \\ Dr Subotica 13, 11000 Belgrade, Serbia \\ ${ }^{4}$ Department of Clinical Neuroscience, Karolinska Institutet, CMM L8:01, 17176 Stockholm, Sweden
}

(Received 30 October 2015; accepted 2 March 2016; published online 23 March 2016)

\begin{abstract}
Dynamic properties of a nonlinear five-dimensional stoichiometric model of the hypothalamicpituitary-adrenal (HPA) axis were systematically investigated. Conditions under which qualitative transitions between dynamic states occur are determined by independently varying the rate constants of all reactions that constitute the model. Bifurcation types were further characterized using continuation algorithms and scale factor methods. Regions of bistability and transitions through supercritical Andronov-Hopf and saddle loop bifurcations were identified. Dynamic state analysis predicts that the HPA axis operates under basal (healthy) physiological conditions close to an Andronov-Hopf bifurcation. Dynamic properties of the stress-control axis have not been characterized experimentally, but modelling suggests that the proximity to a supercritical Andronov-Hopf bifurcation can give the HPA axis both, flexibility to respond to external stimuli and adjust to new conditions and stability, i.e., the capacity to return to the original dynamic state afterwards, which is essential for maintaining homeostasis. The analysis presented here reflects the properties of a low-dimensional model that succinctly describes neurochemical transformations underlying the HPA axis. However, the model accounts correctly for a number of experimentally observed properties of the stress-response axis. We therefore regard that the presented analysis is meaningful, showing how in silico investigations can be used to guide the experimentalists in understanding how the HPA axis activity changes under chronic disease and/or specific pharmacological manipulations. @ 2016 AIP Publishing LLC. [http://dx.doi.org/10.1063/1.4944040]
\end{abstract}

Stress is a physiologic reaction by an organism to an externally imposed demand for change. It manifests itself through the release of a flood of hormones, including cortisol, in the blood circulation, which rouse our body for action. These changes occur through numerous molecular interactions that take place in distinct parts of our body, most notably in several brain regions, the pituitary and adrenal glands. Through these molecular interactions, a vast network of interaction is formed that is known as the Hypothalamic-Pituitary-Adrenal (HPA) axis. Normally, stress is beneficial and helps us to stay focused, attentive, and active, but when persistent and exceeding an individual's threshold, stress stops being helpful and starts causing major damage to our mind and body. Despite intensive research, we still do not understand fully how this extensive network of interactions is controlled and how this protective mechanism, whose main function is to respond to stimuli while maintaining the normal physiologic balance, turns into its opposite and becomes harmful. In this study, we use mathematical modelling and numerical simulations to examine how the dynamics of this complex network is controlled in humans. In order to accomplish this, we have developed a stoichiometric model to concisely describe neurochemical transformations that occur in the HPA

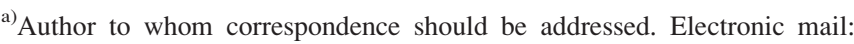
zcupic@nanosys.ihtm.bg.ac.rs. axis. Stoichiometry is a branch in chemistry that quantitatively describes the relationships between reactants and products in a chemical reaction. In a stoichiometric model of a biological system, biochemical pathways are described by stoichiometric relations. In this representation, substances that initiate, i.e., enter a pathway are regarded to behave as reactants, substances that are generated in a pathway are regarded to behave as products, and the rates at which products of a pathway appear are jointly proportional to the amounts of the reactants. In this way, a mathematical framework is developed to describe on chemical and kinetic basis the integration of biochemical pathways that constitute the HPA axis. Such model allows us to describe the changes in the concentration of implicated biomolecules in the form of a system of ordinary differential equations (ODEs) and to use numerical simulations to investigate how the underlying biochemical pathways are intertwined to give an integral HPA axis response at the organism level. By examining theoretically how dynamic properties of the HPA axis are changing when the rates of individual pathways change, we can help the experimentalists to understand how the stress-response is changed in chronic diseases or under the effect of drugs, such as systemically used corticosteroid medications commonly employed to decrease inflammation and reduce the activity of the immune system, or statins, commonly used to reduce blood cholesterol levels. Giving that the HPA axis is a 
nonlinear dynamical network, such behaviour cannot be intuitively predicted. We show in our work how important dynamic properties of the HPA axis are-functioning in the close vicinity to an Andronov-Hopf (AH) bifurcation provides the advantage of fast and controlled response to stress. It gives living organisms the necessary flexibility to adequately respond to stimuli from the surroundings while maintaining their internal balance. Departure from this state results in the reduction of dynamic control and the organism's response to environmental challenge becomes inadequate (too fast, too slow, too strong, or too weak). Mathematical modelling suggests that this may be one way how stress turns into distress.

\section{INTRODUCTION}

Living organisms tightly control the concentration of bioactive molecules and their capacity to uphold specific dynamic states, such as stable steady-states, regular sustained, and mixed-mode oscillations; intermittency or chaos is essential for proper biological functioning. ${ }^{1-14}$ The HPA axis is a dynamical integrator of the nervous and endocrine systems' functions that is of vital importance for maintaining homeostasis in mammalian organisms under normal physiological conditions and under stress. ${ }^{8,9}$ This nonlinear system is characterised by a complex oscillatory dynamics of steroid and peptide hormones with two principal frequencies-ultradian oscillations with a period of 20-120 min that are superimposed on circadian oscillations with a period of about $24 \mathrm{~h} .^{10-12}$ Mechanisms giving rise to ultradian oscillations remain still elusive. Targeted experimental studies have for many years been unsuccessful in identifying the anatomical origin of ultradian rhythms. ${ }^{15}$ However, recent work by the Lightman group suggests that an additional level of glucocorticoid autoregulation may exist within adrenal glands, ${ }^{16}$ further strengthening the view that birhythmic oscillatory changes in blood glucocorticoid levels reflect the integrated activity of pulsatile hypothalamic forcing on an endogenously rhythmic pituitary-adrenal system. The ultradian oscillatory dynamics in HPA axis was previously described in several models assuming different feedback mechanisms, ${ }^{17-35}$ most recently reviewed by Hosseinichimeh et al., ${ }^{36}$ and also in a five-dimensional nonlinear stoichiometric model $^{37}$ where we used instability criteria ${ }^{38-40}$ derived by stoichiometric network analysis (SNA) ${ }^{41,42}$ to identify the narrow range of kinetic parameters (i.e., rate constants) where oscillations in hormone concentrations occur in physiologically relevant levels. In this model, ultradian oscillations arise spontaneously, as a consequence of nonlinear interactions between reactive species, most notably ones that comprise the feedback loop at the pituitary-adrenal level, and the diurnal regulation is dictated externally through a periodic forcing function that imitates the circadian clock synchronized to the daily cycle of light and darkness. ${ }^{38}$ Consequently, in the absence of the external forcing function, an ultradian rhythm at the minute/hour timescale in adrenocorticotropic hormone (ACTH) and cortisol concentration is observed in the model, while it is not observed in corticotropin-releasing hormone (CRH) (see Fig. 2 in Ref. 38), in line with the experimental studies. ${ }^{15}$ In the literature related to mathematical modelling of HPA axis dynamics, endogeneity of ultradian oscillations has been regarded as a dominant and practically unanimously accepted stance (see Table I in Ref. 36).

For a faithful characterization of the repertoire of dynamical states available in the HPA axis, both circadian and (on them superimposed) ultradian oscillations should be included in the models of HPA dynamics. However, depending on the research goal, some models exclude from their description the lower-frequency circadian oscillations ${ }^{18,29,30}$ when focusing on processes that take place at shorter time scales. Similarly, higher-frequency ultradian oscillations may be omitted when processes of interest occur at longer time scales.

In this paper, we focus on the analysis of ultradian oscillations employing our five-dimensional stoichiometric model of the HPA axis, ${ }^{37}$ without incorporating the external forcing function that describes the coupling with circadian oscillations. We systematically analyze conditions under which ultradian oscillations arise or disappear, characterize dynamical states inherent to the system and the manner of their transformation, and investigate the impact that rates of individual reactions between key chemical species included in the model exert on these dynamical states. In particular, we present a more detailed numerical analysis of bifurcations observed in that model, systematically considering the effects of all rate constants one at a time by the method of numerical continuation. $^{43-47}$ Furthermore, some bifurcations are characterized using the analysis of transitions between different dynamic states, comprehensively described by Maselko. ${ }^{48}$

\section{METHODS}

All numerical simulations were executed in MATLAB by employing the ode15s solver based on the Gear algorithm for integration of stiff differential equations. ${ }^{49}$ In all simulations, absolute and relative tolerance errors were $1 \times 10^{-17}$ and $1 \times 10^{-14}$, respectively. Values of kinetic rate constants $\left(\mathrm{k}_{i}\right.$, $i=1-13)$ were as in Table I, unless otherwise stated. If not differently emphasized, the initial conditions for integration of the model's ODE equations were in all numerical simulations as follows: $x_{1,0}=3.4 \times 10^{-4} \mathrm{M}, x_{2,0}=1.0 \times 10^{-12} \mathrm{M}, x_{3,0}=8.0$ $\times 10^{-8} \mathrm{M}, x_{4,0}=4.0 \times 10^{-8}$, and $x_{5,0}=1.5 \times 10^{-9} \mathrm{M}$, where $x_{1,0}, x_{2,0}, x_{3,0}, x_{4,0}$, and $x_{5,0}$, stand for initial concentration values of intermediary chemical species of the model, cholesterol, $\mathrm{CRH}, \mathrm{ACTH}$, cortisol, and aldosterone, respectively.

Bifurcation analysis was carried out by the pseudo-arc length continuation,$^{50}$ which is based on tracing evolution of the steady-state concentration with variation of the control parameter.

\section{MODEL}

As shown in our previous paper, ${ }^{37}$ the dynamics of ultradian HPA oscillations can be faithfully described by 13 reaction steps that concisely represent nonlinear interactions between the 5 most important dynamic variables: cholesterol $\left(\mathrm{X}_{1}\right), \mathrm{CRH}\left(\mathrm{X}_{2}\right)$, ACTH $\left(\mathrm{X}_{3}\right)$, cortisol $\left(\mathrm{X}_{4}\right)$, and aldosterone $\left(\mathrm{X}_{5}\right)$ (Table I). In this model, stoichiometric relations are used to describe the complex neurochemical transformations underlying the HPA axis. Reaction steps (R1), (R2), and (R3) 
TABLE I. Model of the hypothalamic-pituitary-adrenal (HPA) axis.

\begin{tabular}{|c|c|c|c|}
\hline$\stackrel{\mathrm{k}_{1}}{\rightarrow} \mathrm{X}_{1}$ & $v_{1}=\mathrm{k}_{1}$ & $\mathrm{k}_{1}=1.380 \times 10^{-4} \mathrm{M} \mathrm{min}^{-1}$ & $(\mathrm{R} 1)$ \\
\hline$\stackrel{\mathrm{k}_{2}}{\longrightarrow} \mathrm{X}_{2}$ & $v_{2}=\mathrm{k}_{2}$ & $\mathrm{k}_{2}=1.830 \times 10^{-8} \mathrm{M} \mathrm{min}^{-1}$ & $(\mathrm{R} 2)$ \\
\hline$\stackrel{\mathrm{k}_{3}}{\rightarrow} \mathrm{X}_{5}$ & $v_{3}=\mathrm{k}_{3}$ & $\mathrm{k}_{3}=6.090 \times 10^{-11} \mathrm{M} \mathrm{min}^{-1}$ & (R3) \\
\hline$X_{2} \stackrel{\mathrm{k}_{4}}{\rightarrow} \mathrm{X}_{3}$ & $v_{4}=\mathrm{k}_{4} x_{2}$ & $\mathrm{k}_{4}=1.830 \times 10^{4} \mathrm{~min}^{-1}$ & (R4) \\
\hline$X_{1}+X_{3} \stackrel{k_{5}}{\rightarrow} X_{4}$ & $v_{5}=\mathrm{k}_{5} x_{1} x_{3}$ & $\mathrm{k}_{5}=11.94 \mathrm{M}^{-1} \min ^{-1}$ & (R5) \\
\hline$X_{1}+X_{3} \stackrel{k_{6}}{\rightarrow} X_{5}$ & $v_{6}=\mathrm{k}_{6} x_{1} x_{3}$ & $\mathrm{k}_{6}=9.552 \times 10^{-2} \mathrm{M}^{-1} \min ^{-1}$ & (R6) \\
\hline $\mathrm{X}_{3}+2 \mathrm{X}_{4} \stackrel{\mathrm{k}_{7}}{\rightarrow} 3 \mathrm{X}_{4}$ & $v_{7}=\mathrm{k}_{7} x_{3} x_{4}^{2}$ & $\mathrm{k}_{7}=1.260 \times 10^{14} \mathrm{M}^{-2} \min ^{-1}$ & (R7) \\
\hline$X_{5}+2 X_{4} \stackrel{k_{8}}{\rightarrow} X_{4}$ & $v_{8}=\mathrm{k}_{8} x_{5} x_{4}^{2}$ & $\mathrm{k}_{8}=7.050 \times 10^{12} \mathrm{M}^{-2} \mathrm{~min}^{-1}$ & $(\mathrm{R} 8)$ \\
\hline $\mathrm{X}_{1} \stackrel{\mathrm{k}_{9}}{\rightarrow}$ & $v_{9}=\mathrm{k}_{9} x_{1}$ & $\mathrm{k}_{9}=4.500 \times 10^{-2} \mathrm{~min}^{-1}$ & (R9) \\
\hline $\mathrm{X}_{2} \stackrel{\mathrm{k}_{10}}{\longrightarrow}$ & $v_{10}=\mathrm{k}_{10} x_{2}$ & $\mathrm{k}_{10}=1.100 \times 10^{-1} \mathrm{~min}^{-1}$ & (R10) \\
\hline $\mathrm{X}_{3} \stackrel{\mathrm{k}_{11}}{\rightarrow}$ & $v_{11}=\mathrm{k}_{11} x_{3}$ & $\mathrm{k}_{11}=5.350 \times 10^{-2} \mathrm{~min}^{-1}$ & $(\mathrm{R} 11)$ \\
\hline $\mathrm{X}_{4} \stackrel{\mathrm{k}_{12}}{\longrightarrow}$ & $v_{12}=\mathrm{k}_{12} x_{4}$ & $\mathrm{k}_{12}=4.100 \times 10^{-1} \min ^{-1}$ & $(\mathrm{R} 12)$ \\
\hline $\mathrm{X}_{5} \stackrel{\mathrm{k}_{13}}{\rightarrow}$ & $v_{13}=\mathrm{k}_{13} x_{5}$ & $\mathrm{k}_{13}=1.350 \times 10^{-1} \mathrm{~min}^{-1}$ & (R13) \\
\hline
\end{tabular}

describe the biosynthesis of cholesterol, $\mathrm{CRH}$, and aldosterone, respectively. ${ }^{51-53}$ Reaction steps (R9)-(R13) describe the elimination of all species. Reaction steps (R4)-(R8) symbolize the main biochemical processes together with feedback effects of cortisol. ${ }^{53-55}$ Particularly, reaction step (R7) describes the complex cortisol-mediated positive feedback (feed-forward) regulation of the HPA axis, whereas reaction step (R8) describes the cortisol-mediated negative feedback regulation of the HPA axis, both found experimentally. ${ }^{56-58}$

The temporal dynamics of this five-dimensional stoichiometric model of HPA axis self-regulation is described by a system of ODE derived from the model's mechanism based on mass action kinetics 59,60

$$
\begin{gathered}
\frac{\mathrm{d} x_{1}}{\mathrm{~d} t}=v_{1}-\left(v_{5}+v_{6}\right)-v_{9}, \\
\frac{\mathrm{d} x_{2}}{\mathrm{~d} t}=v_{2}-\left(v_{4}+v_{10}\right), \\
\frac{\mathrm{d} x_{3}}{\mathrm{~d} t}=v_{4}-\left(v_{5}+v_{6}\right)-v_{7}-v_{11}, \\
\frac{\mathrm{d} x_{4}}{\mathrm{~d} t}=v_{5}+v_{7}-v_{8}-v_{12}, \\
\frac{\mathrm{d} x_{5}}{\mathrm{~d} t}=v_{3}+v_{6}-v_{8}-v_{13},
\end{gathered}
$$

where $x_{n}$ denotes the concentration of species $\mathrm{X}_{n}, n=1-5$, and $v_{m}$ is the reaction rate of reaction step $\mathrm{R}_{m}, m=1-13$ that can be expressed as a function of related rate constant $\mathrm{k}_{m}$ and the concentrations of species taking part in that particular reaction. Expressions for $v_{m}$ are given in Table I.

Solutions of this system of ODEs (Equations (1a)-(1e)) describe the temporal evolution of the concentrations $\left(x_{n}\right)$ of all investigated species $\left(\mathrm{X}_{n}\right)$ for a given set of parameters, which include the rate constants $\left(\mathrm{k}_{m}\right)$ and initial concentrations of reactants $\left(x_{n, 0}\right)$.

\section{NUMERICAL STABILITY ANALYSIS OF THE MODEL}

Dynamic state of the considered model depends on selected values of rate constants. Bifurcation points, i.e., specific values of rate constants where a qualitative change in the dynamic state is observed, e.g., transition from a stable steady state to an oscillating state, need to be identified by bifurcation analysis. At the same time, distinction can be made between steady states of different stability (stable or unstable) and character (node, saddle, focus, etc.). Hence, different types of bifurcations (Andronov-Hopf, saddle node, etc.) can be identified. However, since bifurcations and particular dynamic states are mostly determined by the steady state properties, as a first step of this analysis, steady states need to be identified.

Here, steady state must be identified from five nonlinear algebraic equations

$$
\begin{gathered}
v_{1}-\left(v_{5}+v_{6}\right)-v_{9}=0, \\
v_{2}-\left(v_{4}+v_{10}\right)=0, \\
v_{4}-\left(v_{5}+v_{6}\right)-v_{7}-v_{11}=0, \\
v_{5}+v_{7}-v_{8}-v_{12}=0, \\
v_{3}+v_{6}-v_{8}-v_{13}=0,
\end{gathered}
$$

where reaction rates are nonlinear functions of the steady state concentrations (see also Table II)

$$
\begin{gathered}
v_{1, \mathrm{ss}}=\mathrm{k}_{1}, \\
v_{2, \mathrm{ss}}=\mathrm{k}_{2}, \\
v_{3, \mathrm{ss}}=\mathrm{k}_{3}, \\
v_{4, \mathrm{ss}}=\mathrm{k}_{4} x_{2, \mathrm{ss}}, \\
v_{5, \mathrm{ss}}=\mathrm{k}_{5} x_{1, \mathrm{ss}} x_{3, \mathrm{ss}}, \\
v_{6, \mathrm{ss}}=\mathrm{k}_{6} x_{1, \mathrm{ss}} x_{3, \mathrm{ss}}, \\
v_{7, \mathrm{ss}}=\mathrm{k}_{7} x_{3, \mathrm{ss}} x_{4, \mathrm{ss}}^{2}, \\
v_{8, \mathrm{ss}}=\mathrm{k}_{8} x_{5, \mathrm{ss}} x_{4, \mathrm{ss}}^{2}, \\
v_{9, \mathrm{ss}}=\mathrm{k}_{9} x_{1, \mathrm{ss}}, \\
v_{10, \mathrm{ss}}=\mathrm{k}_{10} x_{2, \mathrm{ss}}, \\
v_{11, \mathrm{ss}}=\mathrm{k}_{11} x_{3, \mathrm{ss}}, \\
v_{12, \mathrm{ss}}=\mathrm{k}_{12} x_{4, \mathrm{ss}}, \\
v_{13, \mathrm{ss}}=\mathrm{k}_{13} x_{5, \mathrm{ss}} .
\end{gathered}
$$

A system of ODE of this complexity cannot be solved analytically, but it is a more or less an easy task to find numerical solution for an arbitrary set of selected rate constants.

The character of the steady state is evaluated from the eigenvalues of the corresponding Jacoby matrix

$$
\mathbf{J}=\left[\begin{array}{lllll}
\frac{\partial \dot{x}_{1}}{\partial x_{1}} & \frac{\partial \dot{x}_{1}}{\partial x_{2}} & \frac{\partial \dot{x}_{1}}{\partial x_{3}} & \frac{\partial \dot{x}_{1}}{\partial x_{4}} & \frac{\partial \dot{x}_{1}}{\partial x_{5}} \\
\frac{\partial \dot{x}_{2}}{\partial x_{1}} & \frac{\partial \dot{x}_{2}}{\partial x_{2}} & \frac{\partial \dot{x}_{2}}{\partial x_{3}} & \frac{\partial \dot{x}_{2}}{\partial x_{4}} & \frac{\partial \dot{x}_{2}}{\partial x_{5}} \\
\frac{\partial \dot{x}_{3}}{\partial x_{1}} & \frac{\partial \dot{x}_{3}}{\partial x_{2}} & \frac{\partial \dot{x}_{3}}{\partial x_{3}} & \frac{\partial \dot{x}_{3}}{\partial x_{4}} & \frac{\partial \dot{x}_{3}}{\partial x_{5}} \\
\frac{\partial \dot{x}_{4}}{\partial x_{1}} & \frac{\partial \dot{x}_{4}}{\partial x_{2}} & \frac{\partial \dot{x}_{4}}{\partial x_{3}} & \frac{\partial \dot{x}_{4}}{\partial x_{4}} & \frac{\partial \dot{x}_{4}}{\partial x_{5}} \\
\frac{\partial \dot{x}_{5}}{\partial x_{1}} & \frac{\partial \dot{x}_{5}}{\partial x_{2}} & \frac{\partial \dot{x}_{5}}{\partial x_{3}} & \frac{\partial \dot{x}_{5}}{\partial x_{4}} & \frac{\partial \dot{x}_{5}}{\partial x_{5}}
\end{array}\right],
$$


TABLE II. Values of the rate constants at which oscillations appear (lower critical value) and disappear (upper critical value).

\begin{tabular}{lcc}
\hline \hline $\begin{array}{l}\text { Rate } \\
\text { constants }\end{array}$ & $\begin{array}{l}\text { Lower critical value } \\
\text { (type of bifurcation) }\end{array}$ & $\begin{array}{c}\text { Upper critical value } \\
\text { (type of bifurcation) }\end{array}$ \\
\hline$k_{1}$ & $1.42521 \times 10^{-5}(\mathrm{SL})$ & $1.4492 \times 10^{-4}(\mathrm{SAH})$ \\
$k_{2}$ & $1.6123 \times 10^{-8}(\mathrm{SAH})$ & $1.9440 \times 10^{-8}(\mathrm{SAH})$ \\
$k_{3}$ & $0^{\mathrm{a}}$ & $1.2974 \times 10^{-8}(\mathrm{SAH})$ \\
$k_{4}$ & $8.1500 \times 10^{-1}(\mathrm{SAH})$ & $+\infty^{\mathrm{b}}$ \\
$k_{5}$ & $1.2495(\mathrm{SL})$ & $1.2343 \times 10^{1}(\mathrm{SAH})$ \\
$k_{6}$ & $0^{\mathrm{a}}$ & $2.8701(\mathrm{SAH})$ \\
$k_{7}$ & $9.1526 \times 10^{13}(\mathrm{SAH})$ & $1.4467 \times 10^{14}(\mathrm{SAH})$ \\
$k_{8}$ & $0^{\mathrm{a}}$ & $+\infty^{\mathrm{b}}$ \\
$k_{9}$ & $4.2841 \times 10^{-2}(\mathrm{SAH})$ & $4.3575 \times 10^{-1}(\mathrm{SL})$ \\
$k_{10}$ & $0^{\mathrm{a}}$ & $2.4699 \times 10^{3}(\mathrm{SAH})$ \\
$k_{11}$ & $0^{\mathrm{a}}$ & $6.3039 \times 10^{-2}(\mathrm{SAH})$ \\
$k_{12}$ & $3.9739 \times 10^{-1}(\mathrm{SAH})$ & $5.1733 \times 10^{-1}(\mathrm{SAH})$ \\
$k_{13}$ & $0^{\mathrm{a}}$ & $+\infty^{\mathrm{b}}$ \\
\hline \hline
\end{tabular}

${ }^{a}$ The value zero is given as the lower limit of the physically meaningful range of rate constant values and does not correspond to the actual value at the bifurcation point.

${ }^{\mathrm{b}}$ The value $+\infty$ is given here to indicate that an upper bifurcation point was not found. Values up to $1 \times 10^{150}$ were tested in numerical simulations.

where $\dot{x}_{i}=\frac{\mathrm{d} x_{i}}{\mathrm{~d} t}$, and all partial derivatives are calculated using the concentrations in specified steady state. If any eigenvalue of the Jacoby matrix (4) has a positive real part, the steady state is unstable. Conversely, if all eigenvalues have negative real parts, it is stable. ${ }^{38-40}$ The same results are obtained when a somewhat different form of the linearized operator is derived by stoichiometric network analysis. ${ }^{41,42}$ In the present paper, Equation (4) is used for numerical evaluation only.

\section{RESULTS AND DISCUSSIONS}

\section{A. Numerical simulations}

Solution of the system of ODE (1.a-1.e) obtained by numerical integration gives the temporal evolution of the concentrations of species $\mathrm{X}_{1}-\mathrm{X}_{5}$. Under conditions considered here, the concentrations of all species except CRH change oscillatory, with a period of about $25 \mathrm{~min}$. In Fig. 1, Cortisol $\left(\mathrm{X}_{4}\right)$ oscillations are shown as an example. Bifurcation analysis was performed in order to identify in the parameter space conditions under which qualitative transitions in the HPA axis dynamics occur.

\section{B. Bifurcation analysis}

In order to investigate the contribution of individual reaction steps to the overall dynamics and characterize bifurcation types in the model, two methods were applied: the method of numerical continuation ${ }^{43-47}$ and the method proposed by Maselko ${ }^{48}$ and other authors ${ }^{61-75}$ for examination of bifurcations in oscillatory chemical reaction systems. The numerical continuation method was applied to each rate constant separately, keeping all other rate constants fixed. From this analysis, limiting values of rate constants at which oscillations appear or disappear were determined (Table II).

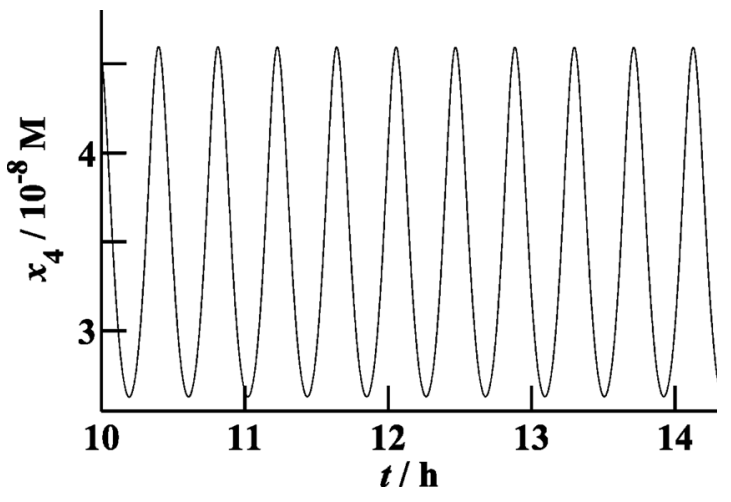

FIG. 1. Time-series showing the temporal dynamics of $\mathrm{X}_{4}$ (cortisol) obtained by numerical integration of ODE (Equations (1a)-(1e)) for the values of kinetic rate constants given in Table I. Time is given in hours and molar concentration in $\mathrm{M}=\mathrm{mol} \mathrm{dm}{ }^{-3}$. (These units were used throughout.)

From Table II, we can see that rate constants can be divided into three groups based on their impact on the system's dynamics. The first group consists of rate constants that have no impact on the emergence of oscillations (at least for the given referent values of other constants). Those rate constants are $\mathrm{k}_{8}$ and $\mathrm{k}_{13}$, and their values can be arbitrarily varied without affecting the appearance or disappearance of oscillatory dynamics. Although these rate constants do not contribute to the emergence of oscillations, they have considerable influence on hormone levels, shape of oscillations, correct oscillatory dynamics, and response to perturbations with endogenous and exogenous compounds (Figure 2).

The second group contains rate constants $\mathrm{k}_{2}, \mathrm{k}_{3}, \mathrm{k}_{4}, \mathrm{k}_{6}$, $\mathrm{k}_{7}, \mathrm{k}_{10}, \mathrm{k}_{11}$, and $\mathrm{k}_{12}$, which all have significant impact on the oscillatory dynamics in the model. By varying these rate constants, transitions between a stable and an unstable steady state were observed and the emergence/disappearance of a stable limit cycle through a supercritical AH (SAH) bifurcation was detected. Bifurcation diagrams for rate constants $\mathrm{k}_{2}$, $\mathrm{k}_{4}, \mathrm{k}_{11}$, and $\mathrm{k}_{12}$ are given in Figure 3. Bifurcation diagrams obtained by varying rate constants $\mathrm{k}_{2}, \mathrm{k}_{7}$, or $\mathrm{k}_{12}$ are structurally identical, showing both lower and upper critical value at which oscillations appear/disappear (Table II, Figs. 3(a) and 3(d)). Bifurcation diagrams obtained by varying rate constants $\mathrm{k}_{3}, \mathrm{k}_{4}, \mathrm{k}_{6}, \mathrm{k}_{10}$, or $\mathrm{k}_{11}$ are structurally similar, having either a lower or an upper critical value (Table II, Figs. 3(b) and 3(c)). The impact of these rate constants was first analysed by numerical continuation methods, by which $\mathrm{AH}$ bifurcations were detected. The critical value at which the AH bifurcation occurs was determined as a point at which a pair of purely imaginary eigenvalues appeared in the Jacoby matrix (Equation (4)).

In order to determine if the AH bifurcation is supercriti$\mathrm{cal} /$ subcritical, we examined how the oscillation amplitudes change as a function of the rate constant value. ${ }^{40}$ Thus, for example, the linear relationship between the square of the amplitude of small-amplitude limit cycle oscillations $\left(\mathrm{A}^{2}\right)$ and rate constant $\left(\mathrm{k}_{2}\right)$ as the control parameter in the vicinity of the lower critical value, i.e., lower bifurcation point, is a proof that a SAH bifurcation is observed (Fig. 4(a)). By extrapolation of this interdependence to zero amplitude, the 

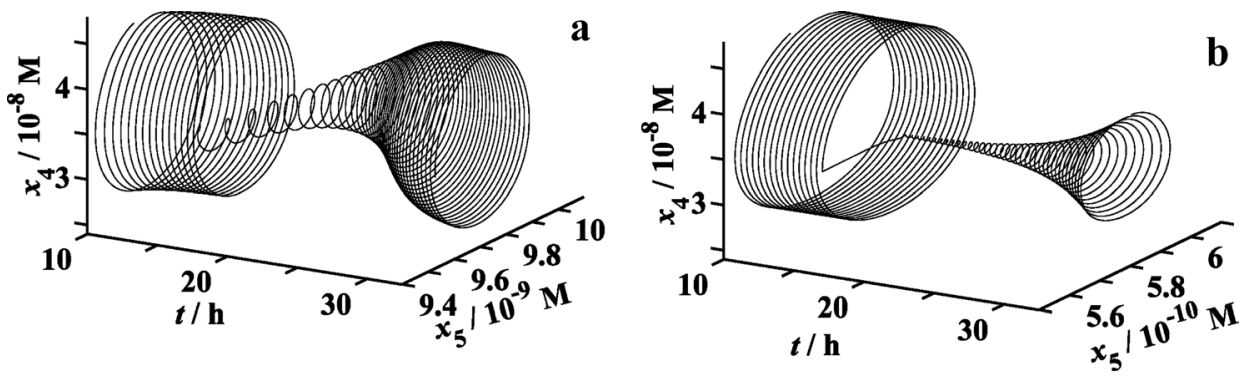

FIG. 2. Different small limit cycle oscillations and distinct responses to perturbations performed by addition of the same amount of $\mathrm{X}_{2}(\mathrm{CRH})$ when different values of the rate constant $\mathrm{k}_{13}$ are used in the HPA axis model: (a) $\mathrm{k}_{13}=0 \min ^{-1}$ and (b) $\mathrm{k}_{13}=1.350$ $\times 10^{-1} \mathrm{~min}^{-1}$. Perturbations were carried out with the same concentration of $\mathrm{X}_{2}, x_{2}=1.2839 \times 10^{-8} \mathrm{M}$.

precise value of the rate constant at the bifurcation point was determined (Table II).

The third group is made of rate constants $\mathrm{k}_{1}, \mathrm{k}_{5}$, and $\mathrm{k}_{9}$, which exert complex impact on the dynamics of the examined model (Fig. 5). The same types of dynamical transitions were observed for all three parameters, but for increasing values of $\mathrm{k}_{1}$ and $\mathrm{k}_{5}$ (exemplified for $\mathrm{k}_{1}$ in Fig. 5(a)) and decreasing values of $k_{9}$ (Fig. 5(b)). As can be seen from Figure 5 , for low values of $\mathrm{k}_{1}$ and high values of $\mathrm{k}_{9}$, three steady states exist simultaneously, two of them being unstable (dashed and dashed-dotted lines) and one being stable (solid line). For low values of $\mathrm{k}_{1}$ or high values of $\mathrm{k}_{9}$, the steady state characterised by the lowest concentration of cortisol $\left(x_{4}\right)$ is a stable node (solid line), for which all eigenvalues of the Jacoby matrix in the steady state (Equation (4)) are negative real numbers. When increasing the value of $\mathrm{k}_{1}$ or decreasing the value of $\mathrm{k}_{9}$, the stable node becomes a stable focus, characterised with two complex-conjugate eigenvalues with negative real parts (dots). At the critical point, this stable focus merges with an unstable steady state and annihilates, giving rise to a saddle with one eigenvalue with a positive real part (dashed line). The steady state with the highest concentration of cortisol $\left(x_{4}\right)$ is a focus. It exists in the whole range of rate constant values and is unstable for low values of $\mathrm{k}_{1}$ or high values of $\mathrm{k}_{9}$, characterised by two complex-conjugate eigenvalues with positive real parts (dashed-dotted line), and stable for high values $\mathrm{k}_{1}$ or low values of $\mathrm{k}_{9}$, characterised by two complex-conjugate eigenvalues with negative real parts (dots).

Multiplicity of steady states leads to emergence of complex bifurcations. In the case of the rate constant $\mathrm{k}_{1}$, in the vicinity of the lower bifurcation point $\left(\mathrm{k}_{1, \mathrm{c} 1}=1.4252 \times 10^{-5} \mathrm{M}\right.$ $\left.\min ^{-1}\right)$, large-amplitude oscillations suddenly appear. The period of the large-amplitude limit cycle oscillations (T) exponentially increases when approaching the bifurcation point $\mathrm{k}_{1, \mathrm{c} 1}$ as evident from the linear dependence of $\mathrm{T}$ on the logarithm of the control parameter $\mathrm{k}_{1}$ expressed in its dimensionless form as $\delta=\left(\mathrm{k}_{1}-\mathrm{k}_{1}, \mathrm{c} 1\right) / \mathrm{k}_{1}, \mathrm{c} 1$ (Figure $\left.4(\mathrm{~b})\right)$. Further analysis showed that the appearance of large-amplitude oscillation depends on the initial conditions. Thus, for example, stable steady state (Figure 4(c)) and sustained largeamplitude oscillations (Figure 4(d)) were obtained for the same value of the control parameter, $\mathrm{k}_{1}=1.42525 \times 10^{-5} \mathrm{M}$ $\min ^{-1}$, but different initial concentrations of cholesterol: $x_{1,0}=1 \times 10^{-4}$ (Figure 4(c)) M, and $x_{1,0}=3.4 \times 10^{-4} \mathrm{M}$ (Figure 4(d)), respectively. Initial concentrations of all other dynamic variables were the same: $x_{2,0}=1.0 \times 10^{-12} \mathrm{M}$, $x_{3,0}=8.0 \times 10^{-8} \mathrm{M}, x_{4,0}=4.0 \times 10^{-8} \mathrm{M}$, and $x_{5,0}=1.5$ $\times 10^{-9} \mathrm{M}$. The fact that the dynamics of the system depends on the initial conditions is a clear indication of multistability,
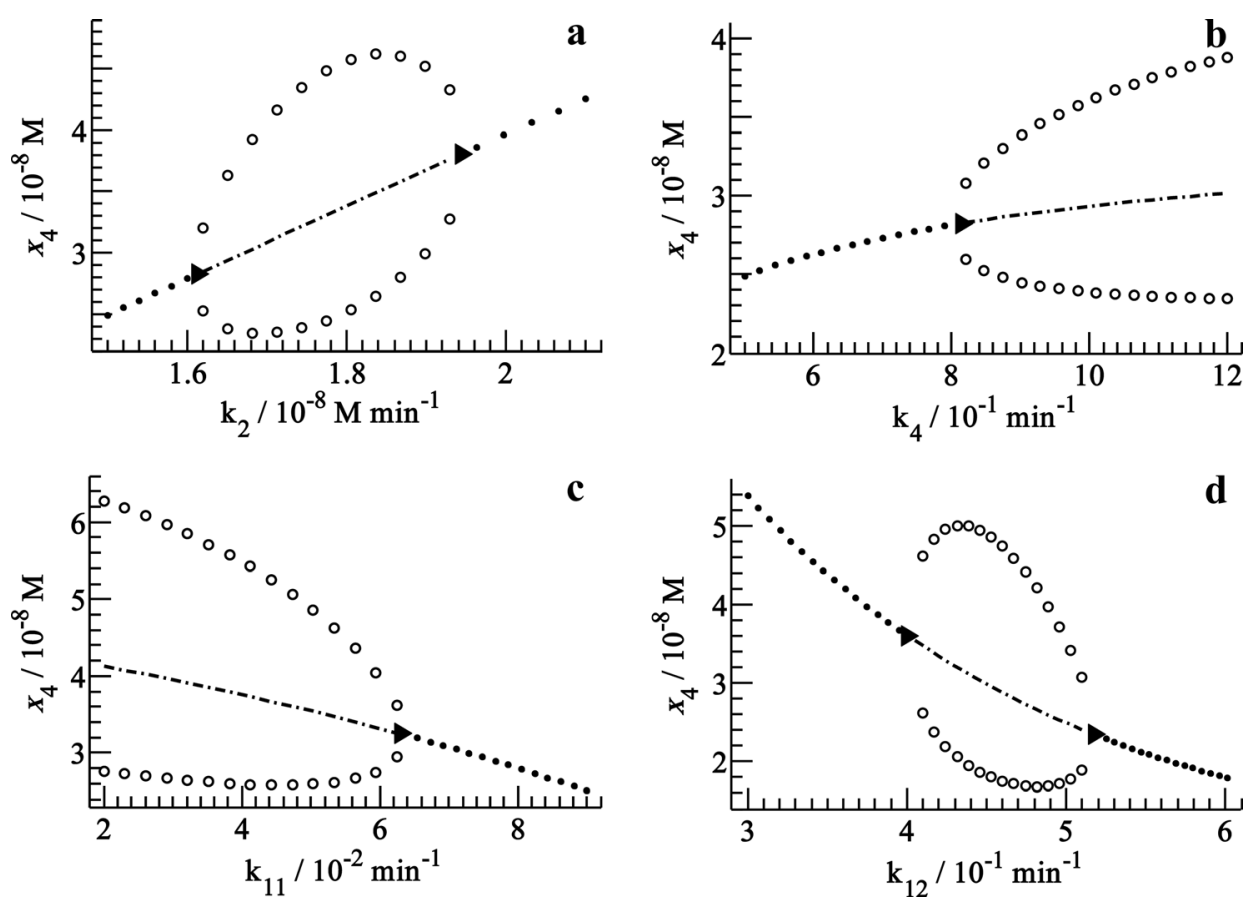

FIG. 3. Bifurcation diagrams showing changes in HPA axis dynamics as a function of the rate constant varied: (a) $\mathrm{k}_{2}$; (b) $\mathrm{k}_{4}$; (c) $\mathrm{k}_{11}$; and (d) $\mathrm{k}_{12}$. Values of all other rate constants were unchanged, as given in Table I. Dashed-dotted lines represent unstable foci. Dots represent stable foci. Oscillatory states are represented by open circles, indicating the minimal and maximal cortisol concentration of sustained oscillations with constant amplitude and frequency, which are established for the indicated value of the control parameter. The exact position of the AH bifurcation point is indicated by the triangle. 

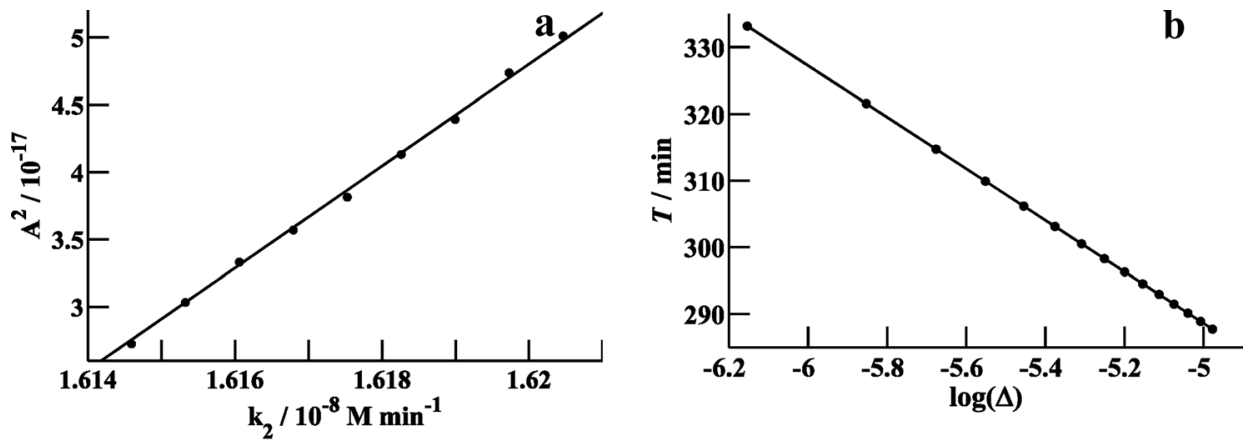

FIG. 4. (a) The square of the amplitude of cortisol oscillations as a function of the control parameter $\mathrm{k}_{2}$ at the vicinity of SAH bifurcation $\quad\left(\mathrm{k}_{2, \mathrm{cl}}=1.6123\right.$ $\times 10^{-8} \mathrm{M} \mathrm{min}^{-1}$ ). (b) Period of largeamplitude oscillations ( $\mathrm{T}$ ) as a function of distance from the SL bifurcation point $\left(\mathrm{k}_{1, \mathrm{c}}=1.42521 \times 10^{-5} \mathrm{M} \mathrm{min}^{-1}\right)$ expressed as the logarithm of the control parameter $\Delta=\left(\mathrm{k}_{1}-\mathrm{k}_{1, \mathrm{c}}\right) / \mathrm{k}_{1, \mathrm{c}}$. (c) Steady state and (d) sustained largeamplitude oscillations obtained for the
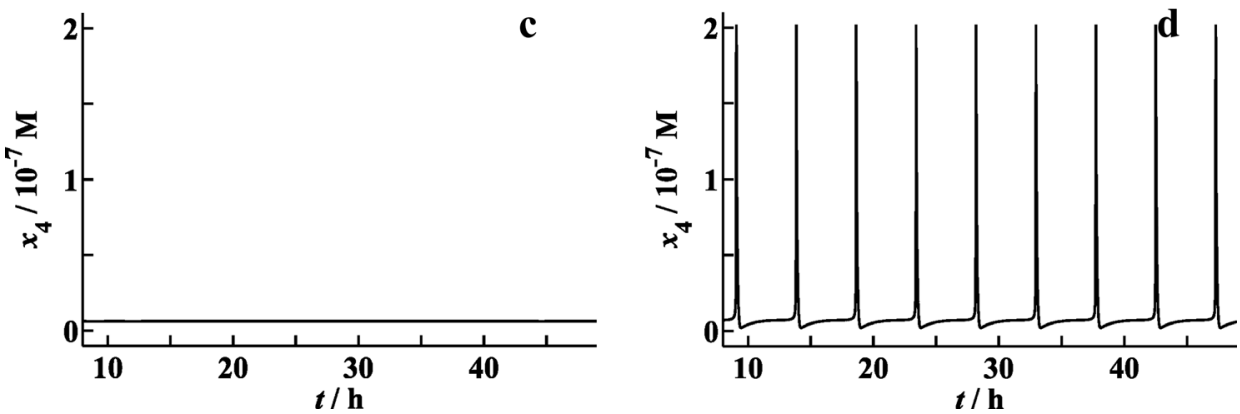
same value of the control parameter $\mathrm{k}_{1}$ $\left(\mathrm{k}_{1}=1.42525 \times 10^{-5} \mathrm{M} \mathrm{min}^{-1}\right)$, but different initial concentrations of cholesterol $\left(x_{1,0}\right): x_{1,0}=1 \times 10^{-4} \mathrm{M}$ in (c) and $x_{1,0}=3.4 \times 10^{-4} \mathrm{M}$ in (d). Initial concentrations of all other dynamic variables were the same: $x_{2,0}=1.0 \times 10^{-12}$ $\mathrm{M}, x_{3,0}=8.0 \times 10^{-8} \mathrm{M}, x_{4,0}=4.0 \times 10^{-8}$ $\mathrm{M}$, and $x_{5,0}=1.5 \times 10^{-9} \mathrm{M}$.

which is also accompanied by hysteresis, typical for a saddle loop (SL) bifurcation where a stable periodic orbit disappears as it becomes a homoclinic loop of a saddle-node. ${ }^{64}$ In the vicinity of the higher bifurcation point, $\mathrm{k}_{1, \mathrm{c} 2}=1.4485 \times 10^{-4} \mathrm{M}$ $\min ^{-1}$, small-amplitude limit cycle oscillations with linear
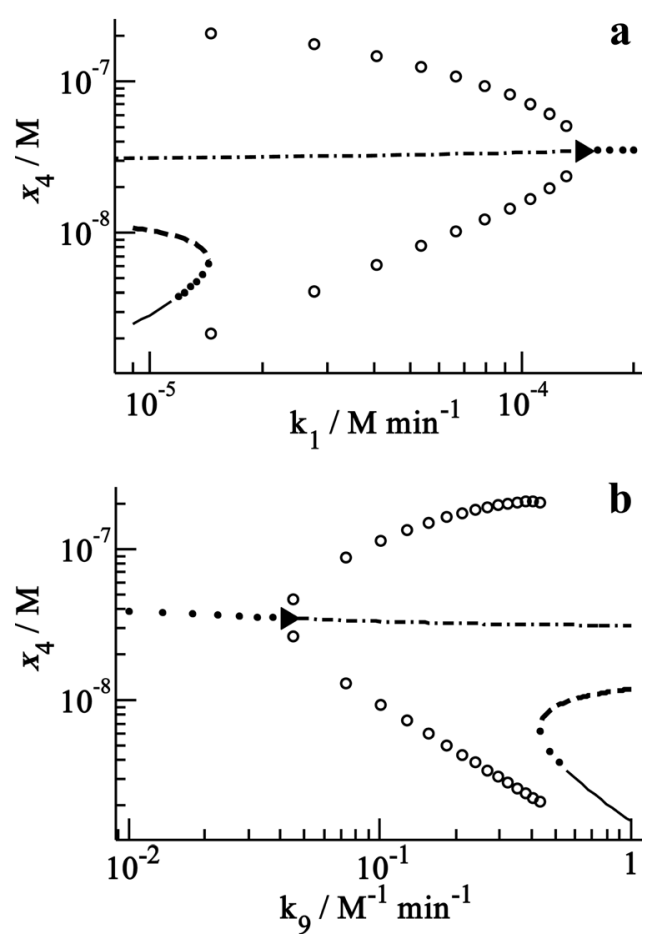

FIG. 5. Bifurcation diagrams showing changes in HPA axis dynamics as a function of the rate constant varied: (a) $\mathrm{k}_{1}$ and (b) $\mathrm{k}_{9}$. Values of all other rate constants were as given in Table I. Solid lines represent stable nodes. Dashed lines represent saddle nodes. Dashed-dotted lines represent unstable foci. Dots represent stable foci. Oscillatory states are represented by open circles, indicating the minimal and maximal cortisol concentration of sustained oscillations with constant amplitude and frequency, which are established for the indicated value of the control parameter. The exact position of the SAH bifurcation point is indicated by the triangle. dependence between the square of the amplitude of smallamplitude limit cycle oscillations and the control parameter were observed. The lack of hysteresis was evident at this point. Thus, we have all proofs that this is a SAH bifurcation. The intercept of $\mathrm{A}^{2}=f\left(\mathrm{k}_{1}\right)$ was obtained at the above mentioned value $\mathrm{k}_{1, \mathrm{c} 2}=1.4485 \times 10^{-4} \mathrm{M} \mathrm{min}^{-1}$. Similar dynamic transition scenarios were previously detected and characterized by Bar-Eli and Brøens in several chemical oscillator models (see, for example, Figure 2(c) in Ref. 75 and references therein). Identical behaviour was also observed in the case of rate constants $\mathrm{k}_{5}$ and $\mathrm{k}_{9}$, except that in the case of $\mathrm{k}_{9}$, saddle-loop was detected at the higher critical value, while $\mathrm{SAH}$ bifurcation was detected at the lower critical value.

\section{Relevance of dynamical transition analysis for applications in biomedicine}

Even though it is well established that HPA axis activity is birhythmic, that dynamic self-regulation is at the core of the neuroendocrine system function, and that dysregulation of HPA axis activity is associated with numerous diseases, ${ }^{80}$ dynamical transitions in this complex system have not been systematically characterised. However, important progress in relating disease states with changes in HPA axis dynamics was made in several recent studies, ${ }^{36,81}$ where it was shown that healthy and disease states may be distinguished through differences in dynamical properties of HPA axis activity. Importance of dynamical transition analysis for understanding disease development in several important rhythmic physiological systems was also underlined in a recent review on dynamical diseases. ${ }^{82}$

The model investigated here suggests that basal (healthy) physiological dynamic state of the HPA axis is close to a SAH bifurcation. ${ }^{37,76}$ While stringent experimental confirmation of this prediction is still awaited, its implications are obviously relevant. The proximity of a SAH bifurcation, with its special geometry of the stable manifold and 
the shape and orientation of the limit cycle, gives the HPA axis exceptional capacity to respond to a challenge and return to its original dynamic state afterwards. Factors that act on the HPA axis can drive it away from the SAH bifurcation and bring the HPA axis towards dynamic states that are less controllable. ${ }^{77}$ For example, in the vicinity of the SL bifurcation, the local geometry is complex and because of bistability the system need not necessarily return to its original dynamic state after a perturbation. From the perspective of efficient homeostasis maintenance, such dynamic structure is clearly unfavourable.

Numerical simulations and dynamical systems theory may be deployed to anticipate dynamical transitions in ultradian HPA axis activity in diseases where the turnover of HPA axis hormones (Fig. 3) or cholesterol (Fig. 5) is altered, or to characterize dynamical transitions in ultradian HPA axis activity induced by medications. For example, glucocorticoids ${ }^{8}$ or statins, ${ }^{78}$ drugs that are widely prescribed in routine medical practice for their anti-inflammatory/ immunosuppressive or cholesterol-level regulating effects, respectively, are representative examples of external perturbators of the HPA axis dynamics. Glucocorticoids are effective in the treatment of many inflammatory conditions, but also as replacement therapy. ${ }^{83}$ However, despite their enormous beneficial effects, glucocorticoid pharmacotherapy remains to be challenging due to, among other issues, the negative side-effects associated with inadequate delivery. ${ }^{84}$ In an effort to moderate these side effects, a portable subcutaneous infusion pump was recently developed to enable complex timing of drug delivery. ${ }^{85}$ Mathematical models that veritably mimic the complex daily dynamics of glucocorticoid secretion, with gradually changing amplitudes of ultradian oscillations during the day, ${ }^{37}$ may be of great value for programming such medical devices to deliver the treatment in accordance with the innate personal rhythm of hormonal secretion that is specific for an individual, leading to prospective clinical applications such as those seen in the field of chronopharmacology. ${ }^{79}$

Furthermore, bifurcation analysis clearly shows how difficult it is to correctly predict changes in HPA axis activity, which is a multidimensional nonlinear dynamical system, by monitoring only one dynamical variable at a single time point during the day, as it is often done in experimental studies and clinical practice. For example, in a recent metaanalysis of randomized placebo-controlled trials on the effects of statins on plasma cortisol concentrations, ${ }^{86}$ it was shown that lipophilic statins (atorvastatin, simvastatin, and lovastatin) increase plasma cortisol concentrations, whereas hydrophilic statins (rosuvastatin and pravastatin) do not, an effect that was allegedly due to a direct action of the tested lipophilic statins on the adrenal gland. While the authors conclude that treatment with hydrophilic statins does not alter plasma cortisol concentrations, implying that HPA axis activity is therefore unaltered by these drugs, our numerical simulations clearly reveal the fallacy of this implication. While mean cortisol concentration remains largely unaltered when cholesterol levels are reduced by cholesterol removal (Fig. 5(b)), changes in ultradian HPA axis activity take indeed place, but in the form of altered amplitude and frequency of ultradian oscillations, and dynamical transitions will occur if cholesterol levels are reduced below the critical point (Fig. 5(b)).

Finally, we would like to underline that bifurcation analysis provides a mathematical framework to quantitatively describe allostasis and measure the allostatic load in a physiological system. The concept of allostasis, introduced in the late 1980s to depict the dynamic process of maintaining functional stability under lasting aversive conditions that drive the physiological system away from its normal functional range, ${ }^{87}$ is often used to describe adaptive transformations in chronic disease states, ${ }^{88,89}$ and the term allostatic load describes the "wear and tear" that a physiological system experiences when undergoing repeated cycles of allostasis. While these concepts are intuitively understandable, they remain impractical, as it is very difficult to assess allostasis and measure the allostatic load. Bifurcation diagrams, like the ones shown in Figs. 3 and 5, clearly show how the HPA axis can self-adjust its underlying dynamics in order to adapt to the effect of an enduring challenge, and the allostatic load in the system can be measured in terms of distance of the actual dynamical state from a bifurcation point. In this way, by introducing concepts from dynamical system theory in neuroendocrinology, one can develop quantitative criteria that objectively characterize states that we can presently describe only subjectively.

\section{ACRONYMS AND NOTATION}

\begin{tabular}{lc}
\hline \hline $\mathrm{CRH}$ & Corticotropin-releasing hormone \\
$\mathrm{ACTH}$ & Adrenocorticotropic hormone \\
$\mathrm{ODE}$ & Ordinary differential equations \\
$x_{n}$ & Concentration of species $\mathrm{X}_{n}$ \\
$\mathrm{k}_{i}$ & Rate constant of the reaction $i$ \\
$v_{i}$ & Rate of the reaction $i$ \\
\hline
\end{tabular}

\section{CONCLUSION}

Dynamic states of ultradian HPA axis activity were examined using a five-dimensional model where the oscillatory states arise from the intrinsic nonlinearity of the underlying biochemical interactions and the entanglement of the investigated species: cholesterol, CRH, ACTH, cortisol, and aldosterone via feedback mechanisms. Numerical continuation algorithms and scaling factor methods developed by Maselko and other authors ${ }^{43-48}$ were applied to the considered model in order to examine its dynamical states. Although applied methods are essentially different, the obtained results are the same.

Bifurcation analysis presented in this paper revealed that rate constants can be classified into three groups based on their impact on the dynamics of the presented model. The first group consists of rate constants $\mathrm{k}_{8}$ and $\mathrm{k}_{13}$, for which it was determined that they do not contribute to the emergence of the Andronov-Hopf bifurcation but they have a big impact on the oscillatory dynamics, as shown in Figure 2. The second group consists of rate constants $\mathrm{k}_{2}, \mathrm{k}_{3}, \mathrm{k}_{4}, \mathrm{k}_{6}, \mathrm{k}_{7}, \mathrm{k}_{10}$, 
$\mathrm{k}_{11}$, and $\mathrm{k}_{12}$, whose varying leads to the appearance of a supercritical Andronov-Hopf bifurcation. The third group consists of rate constants $\mathrm{k}_{1}, \mathrm{k}_{5}$, and $\mathrm{k}_{9}$, whose impact on the model's dynamics is most complex. Varying values of these rate constants can lead to the appearance of two types of bifurcations: supercritical Andronov-Hopf bifurcation and saddle-loop bifurcations. While in the case of the rate constants $\mathrm{k}_{1}$ and $\mathrm{k}_{5}$, supercritical Andronov-Hopf bifurcation appears at the lower critical values and saddle-loop bifurcation appears at the higher critical value, in the case of the rate constant $\mathrm{k}_{9}$, it is the opposite. Here, bistability is identified, indicating the possibility of the HPA axis to acquire either stable oscillations or a stable steady state, depending on initial conditions.

Our work shows that approaches from dynamical systems theory provide an efficient tool for examination of selfregulation in a model of the neuroendocrine system. The work presented here is useful for understanding how chronic deviations induced by internal or external substances affect the HPA axis dynamics and its capacity to respond to stressful stimuli. Furthermore, this work may be useful for understanding how pharmacotherapeutic treatment of chronic conditions influences the HPA axis activity. At present, there are, to our best knowledge, no experimental data that probe the HPA axis dynamics in a systematic way with sufficiently high temporal resolution. The results of our theoretical investigations may therefore be used as a lead for designing such experiments.

\section{ACKNOWLEDGMENTS}

Support from the Karolinska Institute Research Funds, Swedish Research Council, Knut and Alice Wallenberg Foundation, Rajko and Maj Đermanović Fund, CMST COST Action CM1304 "Emergence and Evolution of Complex Chemical Systems," and the Ministry of Education, Science and Technological Development of the Republic of Serbia, Grant Nos. 172015 and 45001, was gratefully acknowledged.

${ }^{1}$ A. Goldbeter, Biochemical Oscillations and Cellular Rhythms (Cambridge University Press, Cambridge, 1996).

${ }^{2}$ I. R. Epstein and J. A. Pojman, An Introduction to Nonlinear Chemical Dynamics: Oscillations, Waves, Patterns, and Chaos: Oscillations, Waves, Patterns, and Chaos (Oxford University Press, Oxford, 1998).

${ }^{3}$ J. Ross, I. Schreiber, and M. O. Vlad, Determination of Complex Reaction Mechanisms: Analysis of Chemical, Biological, and Genetic Networks (Oxford University Press, Oxford, 2006).

${ }^{4}$ A. Hedrih, M. Lazarević, and A. Mitrović-Jovanović, Comput. Biol. Med. 59, 19 (2015).

${ }^{5}$ B. S. Adiwijaya, P. I. Barton, and B. Tidor, Mol. BioSyst. 2, 650 (2006).

${ }^{6}$ S. W. Sowa, M. Baldea, and L. M. Contreras, PLoS Comput. Biol. 10, e1003658 (2014).

${ }^{7}$ O. R. Kols, G. V. Maksimov, and Ch. N. Radenovich, Biophysics of the Rhythmical Excitation (Moscow University Publishing House, Moscow, 1993) (in Russian).

${ }^{8}$ W. Miller and G. Chrousos, in Endocrinol. Metab., edited by P. Felig and L. Frohman (McGraw-Hill, New York, 2001), pp. 387-524.

${ }^{9}$ S. L. Lightman and B. L. Conway-Campbell, Nat. Rev. Neurosci. 11, 710 (2010).

${ }^{10}$ C. Tsigos and G. P. Chrousos, J. Psychosom. Res. 53, 865 (2002).

${ }^{11}$ A. Hartmann, J. D. Veldhuis, M. Deuschle, H. Standhardt, and I. Heuser, Neurobiol. Aging 18, 285 (1997).
${ }^{12}$ A. Gavrila, C.-K. Peng, J. L. Chan, J. E. Mietus, A. L. Goldberger, and C. S. Mantzoros, J. Clin. Endocrinol. Metab. 88, 2838 (2003).

${ }^{13}$ P. O. Westermark and H. Herzel, Cell Rep. 3, 1228 (2013).

${ }^{14}$ A. Korenčič, R. Košir, G. Bordyugov, R. Lehmann, D. Rozman, and H. Herzel, Sci. Rep. 4, 5782 (2014).

${ }^{15}$ J. J. Walker, J. R. Terry, K. Tsaneva-Atanasova, S. P. Armstrong, C. A. McArdle, and S. L. Lightman, J. Neuroendocrinol. 22, 1226 (2010).

${ }^{16}$ J. J. Walker, F. Spiga, R. Gupta, Z. Zhao, S. L. Lightman, and J. R. Terry, J. R. Soc., Interface 12, 20140875 (2015).

${ }^{17}$ D. P. Dempsher, D. S. Gann, and R. D. Phair, Am. J. Physiol. 246, R587 (1984).

${ }^{18}$ Y. Liu, Z.-H. Hu, J.-H. Peng, and B.-Z. Liu, Math. Comput. Modell. 29, 103 (1999).

${ }^{19}$ D. M. Keenan, J. Licinio, and J. D. Veldhuis, Proc. Natl. Acad. Sci. U. S. A. 98, 4028 (2001).

${ }^{20}$ V. Kyrylov, L. A. Severyanova, and A. Vieira, IEEE Trans. Biomed. Eng. 52, 1977 (2005).

${ }^{21}$ Y. Lenbury and P. Pornsawad, Math. Med. Biol. 22, 15 (2005).

${ }^{22}$ S. Jelić, Ž. Čupić, and Lj. Kolar-Anić, Math. Biosci. 197, 173 (2005).

${ }^{23}$ S. Gupta, E. Aslakson, B. M. Gurbaxani, and S. D. Vernon, Theor. Biol. Med. Modell. 4, 8 (2007).

${ }^{24}$ N. Bairagi, S. Chatterjee, and J. Chattopadhyay, Math. Med. Biol. 25, 37 (2008).

${ }^{25}$ M. Conrad, C. Hubold, B. Fischer, and A. Peters, J. Biol. Phys. 35, 149 (2009).

${ }^{26}$ S. Jelić, Ž. Čupić, Lj. Kolar-Anić, and V. Vukojević, Int. J. Nonlinear Sci. Numer. Simul. 10, 1451 (2009).

${ }^{27}$ J. J. Walker, J. R. Terry, and S. L. Lightman, Proc. R. Soc. B 277, 1627 (2010).

${ }^{28}$ F. Vinther, M. Andersen, and J. T. Ottesen, J. Math. Biol. 63, 663 (2011).

${ }^{29}$ V. M. Marković, Ž. Čupić, A. Ivanović, and Lj. Kolar-Anić, Russ. J. Phys. Chem. A 85, 2327 (2011).

${ }^{30}$ S. Maćešić, Ž. Čupić, and Lj. Kolar-Anić, Hem. Ind. 66, 637 (2012).

${ }^{31}$ J. D. Scheff, S. E. Calvano, S. F. Lowry, and I. P. Androulakis, Physiol. Genomics 44, 121 (2012).

${ }^{32}$ S. Postnova, R. Fulcher, H. A. Braun, and P. A. Robinson, Pharmacopsychiatry 46 Suppl 1, S36 (2013).

${ }^{33}$ M. Andersen, F. Vinther, and J. T. Ottesen, Math. Biosci. 246, 122 (2013).

${ }^{34}$ O. A. Moreno-Ramos, M. C. Lattig, and A. F. G. Barrios, Theor. Biol. Med. Modell. 10, 59 (2013).

${ }^{35}$ C. A. Zarzer, M. G. Puchinger, G. Köhler, and P. Kügler, Theor. Biol. Med. Modell. 10, 65 (2013).

${ }^{36}$ N. Hosseinichimeh, H. Rahmandad, and A. K. Wittenborn, Math. Biosci. 268, 52 (2015).

${ }^{37}$ V. M. Marković, Ž. Čupić, S. Maćešić, A. Stanojević, V. Vukojević, and Lj. Kolar-Anić, Math. Med. Biol. 33, 1 (2014).

${ }^{38}$ Ž. Čupić, V. M. Marković, A. Ivanović, and Lj. Kolar-Anić, in Math. Model., edited by C. R. Brennan (Nova Science Publishers, Inc., New York, 2012), pp. 111-178.

${ }^{39}$ Lj. Kolar-Anić, Ž. Čupić, G. Schmitz, and S. Anić, Chem. Eng. Sci. 65, 3718 (2010).

${ }^{40}$ Ž. Čupić, G. Schmitz, and Lj. Kolar- Anić, Ser. A: Appl. Math. Inform. and Mech. 8, 43 (2016).

${ }^{41}$ B. L. Clarke, in Adv. Chem. Phys., edited by I. Prigogine and S. A. Rice (John Wiley \& Sons, Inc., 1980), pp. 1-215.

${ }^{42}$ B. L. Clarke, Cell Biophys. 12, 237 (1988).

${ }^{43}$ E. J. Doedel, W. Govaerts, and Y. A. Kuznetsov, SIAM J. Numer. Anal. 41, 401 (2003).

${ }^{44}$ E. Doedel, H. B. Keller, and J. P. Kernevez, Int. J. Bifurcation Chaos Appl. Sci. Eng. 01, 493 (1991).

${ }^{45}$ J. P. Abbott, Bull. Aust. Math. Soc. 17, 307 (1977).

${ }^{46}$ E. Allgower and K. Georg, Introduction to Numerical Continuation Methods (SIAM, 2003).

${ }^{47}$ A. H. Nayfeh and B. Balachandran, Applied Nonlinear Dynamics: Analytical, Computational and Experimental Methods (John Wiley \& Sons, New York, 1995).

${ }^{48}$ J. Maselko, Chem. Phys. 67, 17 (1982).

${ }^{49}$ C. W. Gear, Numerical Initial Value Problems in Ordinary Differential Equations. Englewood Cliffs (Prentice-Hall, Inc., New Jersey, 1971).

${ }^{50}$ H. B. Keller, in Appl. Bifurc. Theory, edited by P. H. Rabinowitz (Academic Press, 1977), pp. 359-384.

${ }^{51}$ R. A. DeBose-Boyd, Cell Res. 18, 609 (2008).

${ }^{52}$ K. Lu, M.-H. Lee, and S. B. Patel, Trends Endocrinol. Metab. 12, 314 (2001). 
${ }^{53}$ J. Hu, Z. Zhang, W.-J. Shen, and S. Azhar, Nutr. Metab. 7, 47 (2010).

${ }^{54}$ W. L. Miller and R. J. Auchus, Endocr. Rev. 32, 81 (2011).

${ }^{55}$ N. Gallo-Payet and M. D. Payet, Microsc. Res. Tech. 61, 275 (2003).

${ }^{56}$ C. Kellendonk, P. Gass, O. Kretz, G. Schütz, and F. Tronche, Brain Res. Bull. 57, 73 (2002).

${ }^{57}$ J. Schulkin, P. W. Gold, and B. S. McEwen, Psychoneuroendocrinology 23, 219 (1998).

${ }^{58} \mathrm{P}$. Felig, J. D. Baxter, and L. A. Frohman, Endocrinology and Metabolism (McGraw-Hill, New York, 1995).

${ }^{59}$ G. M. Panchenkov and V. P. Lebedev, Chemical Kinetics and Catalysis (MIR, Moscow, 1976).

${ }^{60} \mathrm{~S}$. W. Benson, The Foundation of Chemical Kinetics (McGraw-Hill, Inc., New York, 1960).

${ }^{61}$ J. Maselko and I. R. Epstein, J. Phys. Chem. 88, 5305 (1984).

${ }^{62}$ J. Maselko, M. Alamgir, and I. R. Epstein, Physica D 19, 153 (1986).

${ }^{63}$ Z. Noszticzius, P. Stirling, and M. Wittmann, J. Phys. Chem. 89, 4914 (1985).

${ }^{64}$ V. Gaspar and P. Galambosi, J. Phys. Chem. 90, 2222 (1986).

${ }^{65}$ Z. Noszticzius, M. Wittmann, and P. Stirling, J. Chem. Phys. 86, 1922 (1987).

${ }^{66}$ F. Argoul, A. Arneodo, P. Richetti, and J. C. Roux, J. Chem. Phys. 86, 3325 (1987).

${ }^{67}$ P. Richetti, J. C. Roux, F. Argoul, and A. Arneodo, J. Chem. Phys. 86, 3339 (1987).

${ }^{68}$ V. Gáspár and K. Showalter, J. Chem. Phys. 88, 778 (1988).

${ }^{69}$ C. Kaas-Petersen and S. K. Scott, Physica D 32, 461 (1988).

${ }^{70}$ N. Ganapathisubramanian, J. S. Reckley, and K. Showalter, J. Chem. Phys. 91, 938 (1989).

${ }^{71}$ V. Gáspár, B. Peng, and K. Showalter, in Spat. Inhomogeneities Transient Behav. Chem. Kinet., edited by P. Gray, G. Nicolis, F. Baras, P.
Borckmans, and S. K. Scott (Manchester University Press, Oxford, UK, 1990), pp. 279-289.

${ }^{72}$ P. Gaspard, J. Phys. Chem. 94, 1 (1990).

${ }^{73}$ M. Broens and K. Bar-Eli, J. Phys. Chem. 95, 8706 (1991).

${ }^{74}$ P. E. Strizhak and J. A. Pojman, Chaos 6, 461 (1996).

${ }^{75}$ K. Bar-Eli and M. Broens, J. Phys. Chem. 94, 7170 (1990).

${ }^{76}$ V. M. Marković, Ž. Čupić, V. Vukojević, and Lj. Kolar-Anić, Endocr. J. 58, 889 (2011).

${ }^{77}$ A. E. Motter, Chaos 25, 97621 (2015).

${ }^{78}$ J. L. Goldstein and M. S. Brown, Cell 161, 161 (2015).

${ }^{79}$ R. Dallmann, S. A. Brown, and F. Gachon, Annu. Rev. Pharmacol. Toxicol. 54, 339 (2014).

${ }^{80}$ F. Spiga and S. L. Lightman, Mol. Cell. Endocrinol. 408, 227 (2015).

${ }^{81}$ K. Sriram, M. Rodriguez-Fernandez, and F. J. Doyle III, PLoS Comput. Biol. 8, e1002379 (2012).

${ }^{82}$ L. Glass, Chaos 25, 97603 (2015).

${ }^{83}$ R. A. Overman, J.-Y. Yeh, and C. L. Deal, Arthritis Care Res. (Hoboken) 65, 294 (2013).

${ }^{84}$ G. M. Russell and S. L. Lightman, Expert Opin. Drug Saf. 13, 1501 (2014).

${ }^{85}$ G. M. Russell, C. Durant, A. Ataya, C. Papastathi, R. Bhake, W. Woltersdorf, and S. Lightman, Clin. Endocrinol. (Oxf) 81, 289 (2014).

${ }^{86}$ A. Sahebkar, J. Rathouska, L. E. Simental-Mendía, and P. Nachtigal, Pharmacol. Res. 103, 17 (2016).

${ }^{87} \mathrm{P}$. Sterling and J. Eyer, in Handbook of life stress, cognition and health, edited by S. Fisher and J. Reason (John Wiley \& Sons, Oxford, England, 1988), pp. 629-649.

${ }^{88}$ B. S. McEwen, Neuropsychopharmacology 22, 108 (2000).

${ }^{89}$ G. F. Koob and M. Le Moal, Neuropsychopharmacology 24, 97 (2001). 